RAPIDS

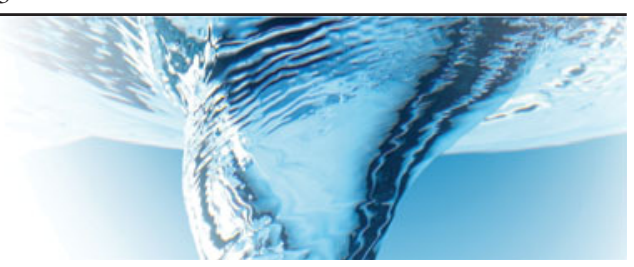

\title{
The interaction of a particle and a polymer brush coating a permeable surface
}

\author{
Avshalom Offner ${ }^{1} \uparrow$ and Guy Z. Ramon ${ }^{1, \dagger}$ \\ ${ }^{1}$ Department of Civil and Environmental Engineering, Technion - Israel Institute of Technology, \\ Haifa 32000, Israel
}

(Received 19 July 2020; revised 4 October 2020; accepted 6 January 2021)

Coating of filtration membranes with a polymer brush holds great promise for efficiently preventing the deposition of fouling particles. The polymer chains are compressed by incoming particles, carried with the permeation flow towards the membrane, and consequently exert a repulsive force that acts to keep the particles away from the membrane surface. Here, we theoretically investigate the effect of a polymer brush coating on the permeation-induced hydrodynamic force, $F_{h}$, pulling a particle towards the membrane, and its balance with the steric repulsion exerted by the compressing brush, resisting the particle's approach. Lubrication theory yields an ordinary differential equation for the pressure, from which $F_{h}$ is calculated numerically. Further, an asymptotic analysis is performed for the limiting cases of a dilute or dense brush, providing analytic expressions that demonstrate how brush properties affect $F_{h}$. Finally, the equilibrium position of a particle is evaluated by considering a balance between the opposing forces. The results provide an upper boundary for the brush properties, beyond which the brush is barely compressed under conditions typical of membrane filtration processes. Further increasing the brush density or thickness only decreases the total system permeance, resulting in increased energy consumption. The results shed light on the mechanisms by which a polymer brush affects the forces acting on a foulant particle, providing quantitative measures for assessing the potential efficacy of brush coatings.

Key words: colloids, lubrication theory

\section{Introduction}

In colloidal science, coating surfaces with a polymer 'brush' is an effective stabilisation strategy, preventing coagulation in suspensions, as was first suggested by

$\dagger$ Email address for correspondence: ramong@technion.ac.il

$\ddagger$ Present address: School of Mathematics, The University of Edinburgh, Edinburgh EH9 3FD, UK.

(C) The Author(s), 2021. Published by Cambridge University Press. This is an Open Access article, distributed under the terms of the Creative Commons Attribution licence (http://creativecommons.org/

licenses/by/4.0/), which permits unrestricted re-use, distribution, and reproduction in any medium, provided the original work is properly cited. 


\section{A. Offner and G.Z. Ramon}

van der Waarden (1950). Polymer brushes are also extensively used as protective layers that resist adsorption of undesired materials onto surfaces (Halperin 1999; Ma et al. 2019; Yan, Bockstaller \& Matyjaszewski 2020). In particular, membranes used for separation processes are prone to unwanted material deposition, exacerbated by the flow through the membranes. Hence, polymer brush coatings have been considered as a promising strategy for fouling prevention (Keating, Imbrogno \& Belfort 2016; Werber, Osuji \& Elimelech 2016). These grafted polymers are known primarily for their ability to exert a repulsive force when compressed (Milner 1989); however, they also affect the hydrodynamic interaction between surfaces. Fredrickson \& Pincus (1991) modelled the effect of a polymer brush on the hydrodynamic force acting on a particle approaching a stationary impermeable surface. Their analysis assumed a highly compressed brush, i.e. a low-permeability brush, modelled as a porous medium squeezed between the two surfaces, for which the flow may be described by the Brinkman equation, employing lubrication theory. In particular, the model assumed that the brush is so dense, translated to an extremely low permeability, that the velocity distribution in it resembles a 'plug flow'. Potanin \& Russel (1995) relaxed this assumption and, using lubrication theory with a full solution of the Brinkman equation, numerically calculated the hydrodynamic force as a function of separation distance and provided analytic expressions for limiting cases. Both Fredrickson \& Pincus (1991) and Potanin \& Russel (1995) showed how the presence of a polymer layer in the thin gap between surfaces significantly increases the hydrodynamic force.

It is well known that the lubrication interaction of a particle in proximity to a planar surface induces a force that may be either attractive or repulsive, depending on the direction of relative motion. Brenner (1961) first solved the case of a particle moving towards a planar impenetrable surface. This result was then extended to permeable surfaces by Goren (1979), demonstrating that surface permeability eliminates the singularity at contact, with the force attaining a constant value that depends on the particle size and the permeability of the surface. Knox et al. (2015) and Venerus (2018) studied the squeeze flow between porous disks, and in the context of membrane separation, most relevant for the present work, this problem was studied by Ramon \& Hoek (2012) and Ramon et al. (2013), who employed a lubrication approach and derived asymptotic solutions for the attractive force between a solid spherical particle and a permeable boundary (further analysis of this problem may be found, for example, in Knox et al. (2017) and in the references listed in Ramon et al. (2013)).

Here, we study the hydrodynamic force on a particle as affected by the presence of a polymer brush coating a permeable surface (e.g. a filtration membrane), and the interplay of this force and the steric repulsion exerted by the brush upon compression. A lubrication model is formulated, from which the pressure field and the force are evaluated. The characteristics of the brush layer and the steric force it exerts are outlined. An asymptotic analysis is performed for various limiting cases. Finally, we consider the equilibrium position of the particle under the opposing action of the two forces.

\section{Model formulation}

We consider a spherical particle with radius $R$ in proximity to a planar membrane, with permeance (permeability per unit thickness) $k$, covered by a polymer brush layer of average thickness $h_{0}$. We assume that there are no chemical reactions between the particle and polymer, and that the polymer chains are of equal length and are in contact with a good solvent. A background pressure $p_{0}$ drives a permeation rate $V_{0}$ through the membrane. The particle, advected by the flow, makes contact with the polymer brush layer, which exerts a 


\section{Interaction of a particle and a polymer brush coating}

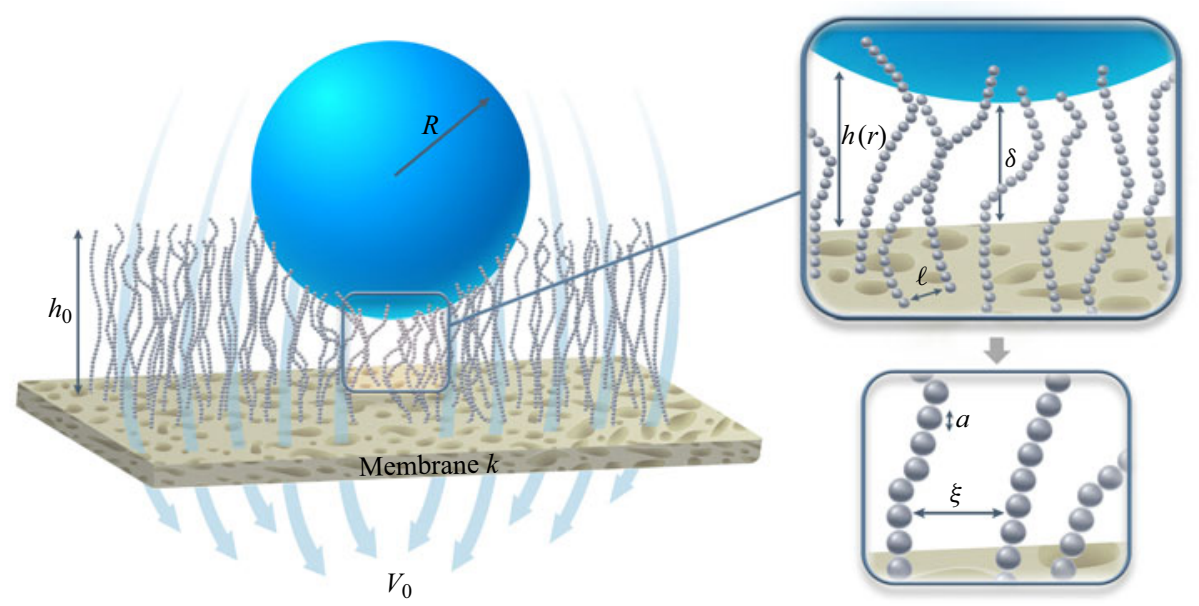

Figure 1. Schematic illustration of the system. A particle of radius $R$ near a membrane (with permeance $k$ ) through which a velocity $V_{0}$ is induced. The particle compresses a polymer brush of initial thickness $h_{0}$. The gap between the particle and the membrane is $h(r) \approx \delta+r^{2} / 2 R$, where $\delta$ is the distance of closest approach. In the diagram, $\ell$ ( $\equiv \Gamma^{-1 / 2}$, where $\Gamma$ is the 'graft density') is the distance between graft points of the polymer at the surface, $a$ is the 'effective' monomer diameter and $\xi$ is the 'correlation length', representing the average distance between chains.

repulsive force as it is compressed, acting to keep the particle away from the membrane. In what follows, we are interested in the attractive hydrodynamic force exerted by the flow and the equilibrium that may exist between it and the repulsive force of the brush as it is compressed (see figure 1 for a schematic illustration).

The hydrodynamic force on the particle, induced by the flow through the brush and the underlying membrane, is calculated in the lubrication limit by integrating the pressure along the particle surface, which is approximated by the shape $h(r)=\delta+r^{2} / 2 R$, where $\delta$ denotes the distance of closest approach. In what follows, a lubrication equation is derived, from which the pressure distribution may be evaluated. We begin with the equations for the steady-state velocity field within the gap confined between the particle and the membrane, in the lubrication limit, namely, the axisymmetric, incompressible continuity and Brinkman equations, given in scaled form as

$$
\begin{gathered}
\frac{1}{r} \frac{\partial}{\partial r}(r u)+\frac{\partial v}{\partial z}=0, \\
\frac{\partial^{2} u}{\partial z^{2}}-\frac{4}{\Lambda \xi(r)^{2}} u=\frac{\mathrm{d} p}{\mathrm{~d} r} .
\end{gathered}
$$

These equations are valid when $h_{0} / R \equiv \varepsilon \ll 1$, where $h_{0} \approx N \Gamma^{1 / 3} a^{5 / 3}$ is the brush thickness at equilibrium (Halperin 1999), with $\Gamma \equiv \ell^{-2}$ denoting the graft density, i.e. the number of polymer chains per unit area, $a$ is the 'effective' monomer size and $N$ is the number of monomers in a chain (see figure 1). Here, $u$ and $v$ are the respective $r$ and $z$ velocity components, scaled by $\varepsilon^{-1} V_{0}$ and $V_{0}$, respectively, and $p$ is the pressure, scaled by $\mu V_{0} R / \varepsilon h_{0}$, where $\mu$ is the viscosity. Further, $r$ is scaled by $\left(2 R h_{0}\right)^{1 / 2}$, while $z, h$ and $\delta$ are scaled by $h_{0}$. The permeability in the Brinkman equations is here represented by the 'correlation length',

$$
\xi(r) \approx \frac{h(r)^{3 / 4}}{(N \Gamma)^{3 / 4} a^{5 / 4}},
$$




\section{A. Offner and G.Z. Ramon}

an important parameter in polymer physics, which characterises the distance over which interactions occur mostly between segments of the same chain (Rubinstein \& Colby 2003). As such, $\xi$ represents an average distance between chains, and so $\xi^{2}$ provides an estimate of the 'pore' dimensions and is used as a proxy of a permeability coefficient (Milner 1989; Fredrickson \& Pincus 1991). With this definition, the brush permeance, $k_{p}$, in dimensionless form, is the parameter $\Lambda \equiv\left(2 \xi_{0} / h_{0}\right)^{2}=4 k_{p} / h_{0}$, where $\xi_{0}=\left.\xi\right|_{h=h_{0}}=$ $\Gamma^{-1 / 2}$ is the characteristic scale for the correlation length.

Potanin \& Russel (1995) derived a solution for $u(r, z)$ by solving $(2.2)$ inside and outside the polymer brush layer, the result of which is mathematically complicated and does not lend itself to derivation of analytic approximations using asymptotic analysis. To facilitate the present analysis, we focus on the 'lubricating region', i.e. a region of scale $\left(R h_{0}\right)^{1 / 2}$ about the point of closest approach, responsible for most of the hydrodynamic interaction. We assume that in this region the particle is in contact with the polymer brush, and hence the velocity is calculated only inside the brush layer. This invariably results, to some extent, in an overestimation of the forces on the particle at large values of $\delta$. With this assumption, the solution to (2.2), subjected to the no-slip boundary conditions, $\left.u\right|_{z=0}=\left.u\right|_{z=h(r)}=0$, is

$$
u(r, z)=-\frac{\Lambda \xi(r)^{2}}{4} \frac{\mathrm{d} p}{\mathrm{~d} r}\left[1-\operatorname{sech}\left(\frac{h(r)}{\Lambda^{1 / 2} \xi(r)}\right) \cosh \left(\frac{h(r)-2 z}{\Lambda^{1 / 2} \xi(r)}\right)\right],
$$

which, when substituted into (2.1) and integrated with respect to $z$, yields the lubrication equation

$$
\frac{\Lambda}{4 r} \frac{\mathrm{d}}{\mathrm{d} r}\left[r h \xi^{2}\left(1-\frac{\tanh \left(h /\left(\Lambda^{1 / 2} \xi\right)\right)}{h /\left(\Lambda^{1 / 2} \xi\right)}\right) \frac{\mathrm{d} p}{\mathrm{~d} r}\right]-\alpha p+1=0,
$$

where $\alpha=2 R k / h_{0}^{2}$. The boundary conditions for (2.5) require that the pressure decays to zero in the far field and that symmetry about the centreline is maintained, i.e.

$$
\left.p\right|_{r \rightarrow \infty}=0,\left.\quad \frac{\mathrm{d} p}{\mathrm{~d} r}\right|_{r=0}=0 .
$$

Finally, the hydrodynamic force is calculated using

$$
F_{h}=2 \pi \int_{0}^{\infty} r p(r) \mathrm{d} r
$$

\subsection{Steric repulsion through polymer brush compression}

The flow driven through the membrane carries particles towards it. However, once a particle makes contact with a brush, which is too dense for it to penetrate (i.e. satisfying $R \gg \xi$ (Halperin 1999)), it compresses the brush, resulting in a repulsive force. Following de Gennes (1980), this force may be evaluated, in the limit $h_{0} \ll R$, as

$$
F_{s}=\int_{A} \Pi \mathrm{d} A=2 \pi R h_{0} \int_{\delta}^{1} \Pi(z) \mathrm{d} z
$$

where $A$ is the area of contact. Here, $\Pi$ is the osmotic pressure, given by the scaling theory of semidilute polymer solutions (Alexander 1977; de Gennes 1980) as

$$
\Pi(z)=k_{B} T \Gamma^{3 / 2}\left(z^{-9 / 4}-z^{3 / 4}\right),
$$




\section{Interaction of a particle and a polymer brush coating}

where $k_{B}$ is Boltzmann's constant and $T$ is the absolute temperature. Combining (2.8) and (2.9), the non-dimensional steric force is (Klein et al. 1994)

$$
F_{s}=7 \delta^{-5 / 4}+5 \delta^{7 / 4}-12,
$$

which is here scaled by $8 \pi R h_{0} k_{B} T \Gamma^{3 / 2} / 35$. The steric force (2.10) holds for $0<\delta \leqslant 1$ and is identically zero when $\delta=1$, i.e. when the particle does not yet compress the brush.

\section{Asymptotic analysis}

Generally, the pressure field provided by (2.5) may only be evaluated numerically. In what follows, we derive analytic solutions for various limiting cases. The scaled permeance of the brush is given by $\Lambda \propto k_{p} / h_{0}$, for which two cases are treated: (i) a 'dilute' brush $(\Lambda \gg 1)$, perturbed near a base state in which the brush is absent; and (ii) a 'dense' brush, for which the base state depends on whether the dominant resistance to flow comes from the brush or the membrane. In the asymptotic analysis, the scaled membrane permeance is small, $\alpha \ll 1$. While the asymptotic solutions provide the pressure field, these are not shown here explicitly since we seek the hydrodynamic force, found by integrating the pressure over the particle surface via (2.7).

\subsection{Dilute brush $(\Lambda \gg 1)$}

In the case of a 'dilute' brush, characterised by a small graft density $\Gamma$, the pressure may be expanded as $p(r, \Lambda)=p_{0}(r)+\Lambda^{-1} p_{1}(r)+O\left(\Lambda^{-2}\right)$, where $p_{0}$ is the pressure field in the absence of a brush. Furthermore,

$$
1-\frac{\tanh \left(h /\left(\Lambda^{1 / 2} \xi\right)\right)}{h /\left(\Lambda^{1 / 2} \xi\right)}=\frac{h^{2}}{3 \Lambda \xi^{2}}-\frac{2 h^{4}}{15 \Lambda^{2} \xi^{4}}+\frac{17 h^{6}}{315 \Lambda^{3} \xi^{6}}+O\left(\Lambda^{-4}\right) .
$$

Including the membrane permeance in this case is achieved by assuming $\alpha \approx \Lambda^{-1}$, leading to a set of perturbation problems, written here to $O\left(\Lambda^{-2}\right)$ as

$$
\frac{1}{12 r} \frac{\mathrm{d}}{\mathrm{d} r}\left[r h^{3} \frac{\mathrm{d} p_{i}}{\mathrm{~d} r}\right]= \begin{cases}-1, & i=0, \\ \Lambda \alpha p_{i-1}+\frac{1}{30 r} \frac{\mathrm{d}}{\mathrm{d} r}\left[r h^{7 / 2} \frac{\mathrm{d} p_{i-1}}{\mathrm{~d} r}\right], & i=1, \\ \Lambda \alpha p_{i-1}+\frac{1}{30 r} \frac{\mathrm{d}}{\mathrm{d} r}\left[r h^{7 / 2} \frac{\mathrm{d} p_{i-1}}{\mathrm{~d} r}\right]-\frac{17}{1260 r} \frac{\mathrm{d}}{\mathrm{d} r}\left[r h^{4} \frac{\mathrm{d} p_{i-2}}{\mathrm{~d} r}\right], & i=2,\end{cases}
$$

in which the term $\alpha \Lambda=O(1)$, with $\alpha \neq \Lambda^{-1}$ necessarily. Three (and more) terms may be calculated for the expansion of $p$; however, only the first two terms yield analytic expressions for the force. The hydrodynamic force in this case is

$$
F_{h}=\frac{3 \pi}{2 \delta}-\frac{3 \pi \alpha}{4 \delta^{3}}+\frac{8 \pi}{5 \Lambda \delta^{1 / 2}}+O\left(\Lambda^{-2}\right)
$$

in which the leading-order term recovers the result of Brenner (1961) for the force on a sphere approaching an impermeable surface. The second and third terms are the $O\left(\Lambda^{-1}\right)$ correction to this classical result, where the second term recovers the result of Goren (1979) and Ramon et al. (2013), showing that a finite permeance decreases the force, and the third term is a new correction representing the addition of the polymer brush on the surface, which increases the force. 


\section{A. Offner and G.Z. Ramon}

\subsection{Dense brush $(\Lambda \ll 1)$}

In the case of a dense brush, the hyperbolic tangent term in (2.5) is approximately $\Lambda^{1 / 2} \xi / h$. If $\Lambda, \alpha \ll 1$, however, the pressure in (2.5) must be rescaled to avoid a singularity in the leading-order lubrication equation. We begin by rescaling the coordinate $r=\delta^{1 / 2} \eta$, to obtain

$$
\frac{\Lambda \delta^{3 / 2}}{4 \eta} \frac{\mathrm{d}}{\mathrm{d} \eta}\left[\eta\left(1+\eta^{2}\right)^{5 / 2}\left(1-\frac{\Lambda^{1 / 2}}{\delta^{1 / 4}\left(1+\eta^{2}\right)^{1 / 4}}\right) \frac{\mathrm{d} p}{\mathrm{~d} \eta}\right]-\alpha p+1=0, \quad \Lambda, \alpha \ll 1 .
$$

Next, we define a new dimensionless parameter,

$$
\beta \equiv \frac{4 \alpha}{\Lambda \delta^{3 / 2}}=\frac{1}{\delta^{3 / 2}} \frac{k}{\varepsilon k_{p}},
$$

and identify two possible scenarios: $\beta \ll 1$, i.e. the membrane permeance is much smaller than that of the polymer brush, $k \ll \varepsilon k_{p}$; and $\beta \gg 1$, for which the permeance ratio between the membrane and brush satisfies $k / k_{p} \gg \varepsilon$.

\subsubsection{The case $\beta \ll 1$}

In this limit the brush layer permeance, however small, is still much larger than that of the membrane it covers. The pressure is rescaled as $P=\Lambda \delta^{3 / 2} p / 4$ and (3.4) becomes

$$
\frac{1}{\eta} \frac{\mathrm{d}}{\mathrm{d} \eta}\left[\eta\left(1+\eta^{2}\right)^{5 / 2}\left(1-\frac{2 \alpha^{1 / 2}}{\delta \beta^{1 / 2}}\left(1+\eta^{2}\right)^{-1 / 4}\right) \frac{\mathrm{d} P}{\mathrm{~d} \eta}\right]-\beta P+1=0 .
$$

The asymptotic order of $\alpha / \beta \propto \Lambda \ll 1$ is indeterminate. The effect of reducing the brush density is found by assuming $\alpha=O\left(\beta^{3}\right)$ and expanding the pressure as $P=P_{0}(\eta)+$ $\beta P_{1}(\eta)+O\left(\beta^{2}\right)$. For convenience, we define

$$
\gamma=\frac{2 \alpha^{1 / 2}}{\delta \beta^{3 / 2}}=O(1)
$$

such that (3.6) may be written as

$$
\frac{1}{\eta} \frac{\mathrm{d}}{\mathrm{d} \eta}\left[\eta\left(1+\eta^{2}\right)^{5 / 2} \frac{\mathrm{d} P_{i}}{\mathrm{~d} r}\right]= \begin{cases}-1, & i=0, \\ P_{i-1}+\frac{\gamma}{\eta} \frac{\mathrm{d}}{\mathrm{d} \eta}\left[\eta\left(1+\eta^{2}\right)^{9 / 4} \frac{\mathrm{d} P_{i-1}}{\mathrm{~d} r}\right], & i \geqslant 1 .\end{cases}
$$

Here, an analytic expression for the force (2.7) may be found only to $O(\beta)$,

$$
F_{h}=\frac{\pi}{\Lambda \delta^{1 / 2}}\left[\frac{4}{3}+\beta\left(\frac{16 \gamma}{21}-\frac{1}{18}\right)\right]+O\left(\beta^{2}\right),
$$

where the leading-order term recovers the 'plug-flow' result derived by Fredrickson \& Pincus (1991), and the $O(\beta)$ term is the correction due to a finite (small) membrane permeance and a small increase in the brush permeance. While the membrane permeance clearly decreases the hydrodynamic force, lowering the brush density - which is expected to decrease this force - is here seen, in the $O(\beta)$ correction, to increase it. This is because, as $\Lambda \rightarrow 0$, the polymer brush becomes impermeable, such that the flow is unaffected by the presence of the membrane. Decreasing the brush density 'exposes' the flow to the membrane, which is then less permeable than the brush. Consequently, the combined permeance (membrane and brush) decreases and the correction to the force is positive. 


\section{Interaction of a particle and a polymer brush coating}

Rewriting (3.9) in terms of $\alpha, \Lambda$ and $\delta$ gives

$$
F_{h}=\frac{4 \pi}{3 \Lambda \delta^{1 / 2}}-\frac{2 \pi \alpha}{9 \Lambda^{2} \delta^{2}}+\frac{16 \pi}{21 \Lambda^{1 / 2} \delta^{3 / 4}}
$$

in which the requirement that the leading-order term dominates over the last term leads to $\delta \gg \Lambda^{2}$ for which the correction is valid.

\subsubsection{The case $\beta \gg 1$}

Here, the permeance ratio is $k / k_{p} \gg \varepsilon$ and the pressure is rescaled as $\mathcal{P}=\alpha p$, so that

$$
\frac{\beta^{-1}}{\eta} \frac{\mathrm{d}}{\mathrm{d} \eta}\left[\eta\left(1+\eta^{2}\right)^{5 / 2}\left(1-\frac{2 \alpha^{1 / 2}}{\delta \beta^{1 / 2}}\left(1+\eta^{2}\right)^{-1 / 4}\right) \frac{\mathrm{d} \mathcal{P}}{\mathrm{d} \eta}\right]-\mathcal{P}+1=0,
$$

in which expanding $\mathcal{P}$ as a series of $\beta^{-1}$ clearly results in a singular perturbation problem, with the leading-order solution $\mathcal{P}_{0}=1$ unable to satisfy the far-field boundary condition. We introduce yet another rescaled coordinate $\eta=\beta^{1 / 3} \zeta$, with which (3.11) becomes

$$
\frac{1}{\zeta} \frac{\mathrm{d}}{\mathrm{d} \zeta}\left[\zeta\left(\beta^{-2 / 3}+\zeta^{2}\right)^{5 / 2}\left(1-\frac{2 \alpha^{1 / 2}}{\delta \beta^{2 / 3}}\left(\beta^{-2 / 3}+\zeta^{2}\right)^{-1 / 4}\right) \frac{\mathrm{d} \mathcal{P}}{\mathrm{d} \zeta}\right]-\mathcal{P}+1=0
$$

enabling a regular perturbation for $\beta^{-2 / 3} \ll 1$ by expanding $\mathcal{P}(\zeta, \beta)=\mathcal{P}_{0}(\zeta)+\beta^{-2 / 3}$ $\mathcal{P}_{1}(\zeta)+O\left(\beta^{-4 / 3}\right)$. An analytic solution is found for the leading-order equation

$$
\frac{1}{\zeta} \frac{\mathrm{d}}{\mathrm{d} \zeta}\left[\zeta^{6} \frac{\mathrm{d} \mathcal{P}_{0}}{\mathrm{~d} \zeta}\right]-\mathcal{P}_{0}+1=0
$$

and the corresponding force, written in term of $\Lambda, \alpha$ and $\delta$, is

$$
F_{h}=\frac{c}{\Lambda^{2 / 3} \alpha^{1 / 3} \delta}
$$

where $c=3^{1 / 6} \Gamma(1 / 3)^{2} / 2^{2 / 3} \approx 5.43$.

The dependence of the hydrodynamic force, $F_{h}$, on the brush permeance, $\Lambda$, is presented in figure 2(a), comparing the asymptotics with the numerical calculations. Figure $2(b)$ maps the asymptotic solutions from the current work, as well as those from previous studies, in the $\Lambda-\beta$ plane. Here, results appearing on the right end of the scale describe the absence of a brush. Meanwhile, $\beta$ marks the permeance ratio between the membrane and brush, such that low values denote a brush permeance much larger than that of the membrane.

\section{Equilibrium}

When the hydrodynamic and steric forces are exactly balanced, the particle reaches its equilibrium position, which should be sufficiently away from the membrane surface so as to avoid performance deterioration (Keating et al. 2016). In order to evaluate this balance, 


\section{A. Offner and G.Z. Ramon}
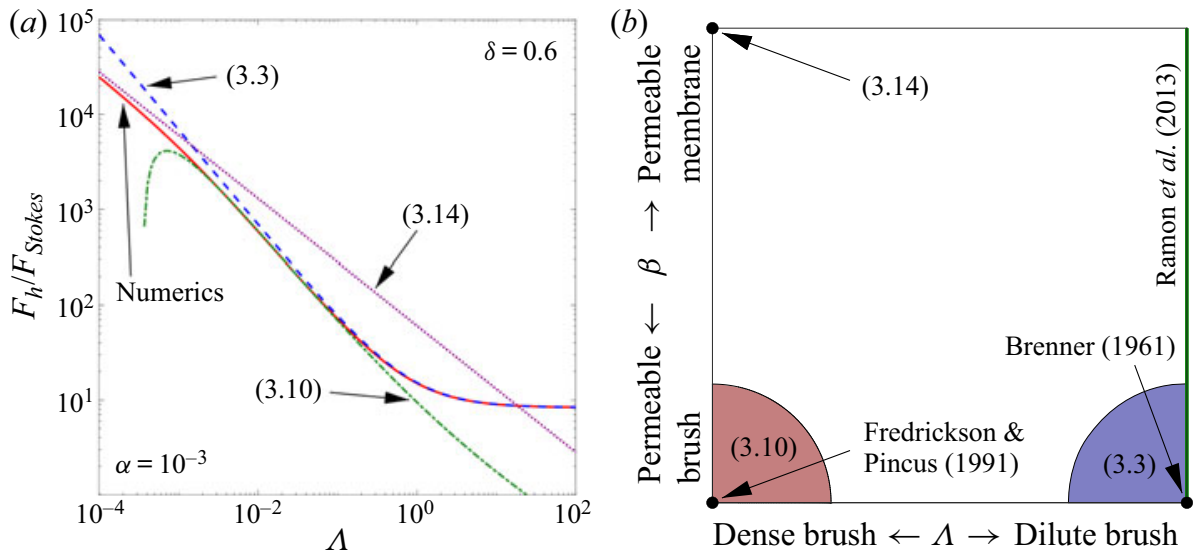

Figure 2. (a) The hydrodynamic force, scaled by $F_{\text {Stokes }}=6 \pi \mu R V_{0}$, as a function of brush permeance, $\Lambda \equiv 4 k_{p} / h_{0}$, for $\alpha=10^{-3}$. The red solid line is the numerical calculation, while the blue dashed, green dashed-dotted and violet dotted lines mark the asymptotic solutions (3.3), (3.10) and (3.14), respectively. (b) Mapping of the asymptotic solutions to (2.5) in the $\Lambda-\beta$ plane. Black dots mark leading-order solutions; lines and shaded areas mark asymptotic solutions valid for a finite range of $\Lambda, \beta$.

$F_{h}$ is rescaled by $8 \pi R h_{0} k_{B} T \Gamma^{3 / 2} / 35$, so that

$$
F_{h}=\hat{V}_{0} \Lambda^{3 / 2} \int_{0}^{\infty} r p(r) \mathrm{d} r,
$$

where the scaled permeation velocity is defined as

$$
\hat{V}_{0}=\frac{35 \mu R h_{0} V_{0}}{8 \pi k_{B} T} .
$$

The asymptotic solutions (3.3), (3.10) and (3.14) are, in rescaled form, respectively,

$$
\begin{gathered}
F_{h}=\frac{\hat{V}_{0}}{2}\left(\frac{3 \Lambda^{3 / 2}}{2 \delta}-\frac{3 \alpha \Lambda^{3 / 2}}{4 \delta^{3}}+\frac{8 \Lambda^{1 / 2}}{5 \delta^{1 / 2}}\right), \quad \Lambda \gg 1, \\
F_{h}=\frac{\hat{V}_{0}}{2}\left(\frac{4 \Lambda^{1 / 2}}{3 \delta^{1 / 2}}-\frac{2 \alpha}{9 \Lambda^{1 / 2} \delta^{2}}+\frac{16 \Lambda}{21 \delta^{3 / 4}}\right), \quad \Lambda, \beta \ll 1, \\
F_{h}=\frac{c^{*} \hat{V}_{0} \Lambda^{5 / 6}}{\alpha^{1 / 3} \delta}, \quad \Lambda \ll 1, \beta \gg 1,
\end{gathered}
$$

where $c^{*}=c / 2 \pi \approx 0.86$. The scaled equilibrium position, $\delta_{e q}$, is found by equating (4.1) with (2.10) and numerically solving for $\delta$. Analytic expressions may be obtained at the limit of close approach, i.e. when $\delta \ll 1$, in which (2.10) simplifies to $F_{s} \approx 7 \delta^{-5 / 4}$. Considering the limit $\alpha \rightarrow 0$ simplifies (4.3) and (4.4), resulting in the following approximations:

$$
\begin{gathered}
\delta_{e q} \approx\left(\frac{14}{3}\right)^{4} \Lambda^{-6} \hat{V}_{0}^{-4}, \quad \delta \ll 1, \Lambda \gg 1, \\
\delta_{e q} \approx\left(\frac{21}{4}\right)^{4 / 3} \Lambda^{-2 / 3} \hat{V}_{0}^{-4 / 3}, \quad \delta \ll 1, \Lambda \ll 1 .
\end{gathered}
$$


(a)

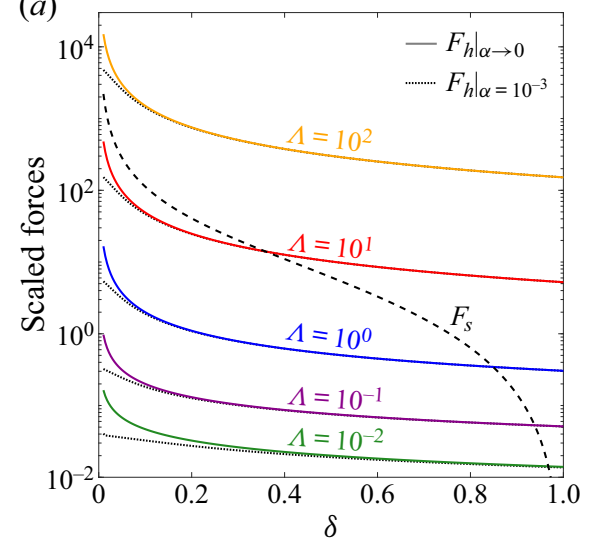

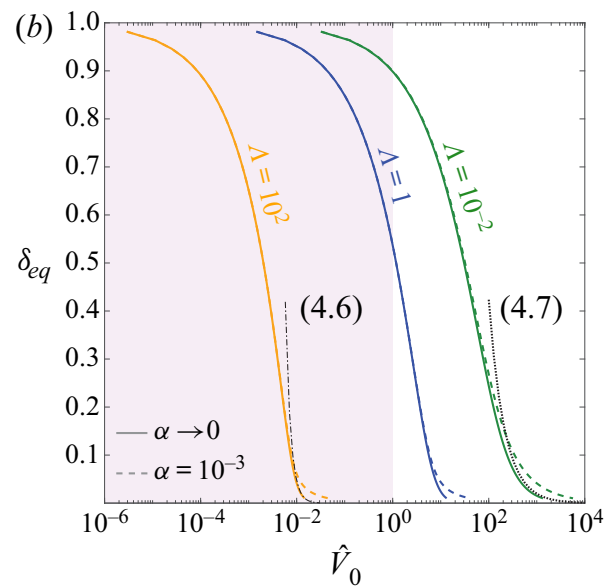

Figure 3. (a) The hydrodynamic ( $F_{h}$; solid and dotted-dashed curves for $\alpha \rightarrow 0$ and $\alpha=10^{-3}$, respectively) and steric $\left(F_{s}\right.$; dashed black curve) forces, evenly scaled, as functions of the scaled distance, $\delta$, for various values of $\Lambda$, the brush permeance. Here, the scaled permeation velocity is $\hat{V}_{0}=10^{-1} . F_{h}$ is calculated numerically by (4.1), and $F_{s}$ is given by (2.10). (b) Equilibrium position, $\delta_{e q}$, for which $F_{h}=F_{s}$, as a function of $\hat{V}_{0}$ for varying $\Lambda$. Solid and dashed lines denote $\alpha \rightarrow 0$ and $\alpha=10^{-3}$, respectively, and the black dashed and dotted curves are the asymptotics for $\delta \ll 1, \Lambda \gg 1$ and $\Lambda \ll 1$, respectively. The shaded area marks the typical range of $\hat{V}_{0}$ for membrane separation (see table 1).

Figure 3(a) illustrates the dependence of each force on the distance from the membrane, $\delta$, at a scaled permeation velocity $\hat{V}_{0}=10^{-1}$, which is within the characteristic range of membrane separation (see table 1), and is chosen for a clear comparison between the competing forces. The hydrodynamic force is calculated numerically by (4.1) for various values of the brush permeance, $\Lambda$, with membrane permeance set as $\alpha \rightarrow 0$ and $\alpha=10^{-3}$. The steric force, $F_{S}(\delta)$, is calculated explicitly from (2.10). Intersections of the curves for $F_{s}$ and $F_{h}$ mark the equilibrium positions at each $\Lambda$. Figure 3(a) demonstrates that the membrane permeance has little effect on the lubrication force for large $\delta$. At close approach, however, $F_{h}$ tends to infinity for $\alpha \rightarrow 0$, while $\alpha>0$ leads to a finite force as $\delta \rightarrow 0$, as expected. We note that here the hydrodynamic force increases with $\Lambda$, as opposed to the trend in figure 2, due to the rescaling of $F_{h}$ in (4.1), to enable an even comparison with $F_{s}$, while the results in figure 2 are scaled with the Stokes drag, in order to emphasise the increased drag due to the lubrication interaction. Physically, a decreased $\Lambda$ means the brush becomes denser and so $F_{h}$ increases. Within the parameter range shown in table 1 , representing 'real-world' membrane separation, a brush permeance of $\Lambda=O\left(10^{-3}\right)$ results in $\delta_{e q} \approx 1$, at which point decreasing $\Lambda$ further will not improve the anti-fouling performance. Rather, it will decrease the combined membrane and brush permeance, $K=\left(k^{-1}+k_{p}^{-1}\right)^{-1}$, which will then require a larger pressure $p_{0}$ to maintain the same permeation velocity, resulting in increased energy consumption.

Figure $3(b)$ displays the equilibrium position of the particle, $\delta_{e q}$, found via the balance between the attractive and repulsive forces, as a function of the scaled velocity, $\hat{V}_{0}$, for representative values of $\Lambda$, with $\alpha \rightarrow 0$ and $\alpha=10^{-3}$. Naturally, an increase in $\hat{V}_{0}$ forces the particle closer to the membrane. A denser brush increases particle repulsion, resulting in a larger $\delta_{e q}$ for a given $\hat{V}_{0}$. Neglecting the effect of membrane permeance $(\alpha \rightarrow 0)$, a decrease in $\Lambda$ shifts the slope from $\delta_{e q} \propto \hat{V}_{0}^{-4}(\Lambda \gg 1)$ to $\delta_{e q} \propto \hat{V}_{0}^{-4 / 3}(\Lambda \ll 1)$, corresponding to the shift from $F_{h} \propto \delta^{-1}$ (Brenner 1961) to $F_{h} \propto \Lambda^{-1} \delta^{-1 / 2}$ (Fredrickson 


\begin{tabular}{lccccccc}
\hline & Description & Unit & Range & & Description & Unit & Range \\
$R$ & Particle radius & $\mu \mathrm{m}$ & $0.1-1$ & $N$ & No. monomers in chain & - & $10^{2}-10^{3}$ \\
$k$ & Membrane permeance & $\mathrm{nm}$ & $10^{-6}-10^{-3}$ & $\Gamma$ & Graft density & $\mathrm{nm}^{-2}$ & $0.01-0.5$ \\
$a$ & Monomer size & $\mathrm{nm}$ & $0.1-1$ & $V_{0}$ & Permeation velocity & $\mu \mathrm{m} \mathrm{s}^{-1}$ & $1-100$
\end{tabular}

Table 1. Model parameters and characteristic values.

\& Pincus 1991), while increasing $\alpha$ always tends to decrease $F_{h}$, as discussed in $\S 3$. Consequently, for a given $\hat{V}_{0}$, the $\delta_{e q}$ curves for $\alpha=10^{-3}$ lie above the corresponding ones for $\alpha \rightarrow 0$. While for $\Lambda \gtrsim 1$ the effect of $\alpha \neq 0$ is seen only at small $\delta_{e q}$, as the brush becomes denser the membrane permeance affects the equilibrium over a wider range. Physically, this is because thickening the brush enhances the resistance to the flow within the thin gap, and therefore the force extends over a longer distance. In the case $\alpha>0$, the decrease in $F_{h}$ then affects a wider range of $\delta$.

\section{Concluding remarks}

In this work we examined the effect of a polymer brush coating, applied to a membrane surface, on the hydrodynamic force, $F_{h}$, acting on a particle at close approach. Asymptotic solutions were derived, providing insight on trends in $F_{h}$ as both the membrane and/or brush permeances, $\alpha$ and $\Lambda$, respectively, are varied. Based on the balance between $F_{h}$ and the steric repulsion force, exerted by the brush as it is compressed by the particle, the equilibrium position of the particle, $\delta_{e q}$, was calculated as a function of the scaled permeation velocity through the membrane, $\hat{V}_{0}$.

Equilibrium calculations illustrate the extent to which increasing $\hat{V}_{0}$ brings the particle closer to the membrane. The results also show that, beyond a certain brush density, one has $\delta_{e q} \rightarrow 1$, whereby any further decrease to $\Lambda$ will only increase the resistance to the flow and result in an increased energy input required to maintain the same permeation rate through the membrane. At close approach $\left(\delta_{e q} \ll 1\right)$, analytic approximations were derived, showing the extent to which a dense brush $(\Lambda \ll 1)$ protects the surface compared with a dilute brush $(\Lambda \gg 1)-$ trending as $\delta_{e q} \propto \hat{V}_{0}^{-4 / 3}$ and $\hat{V}_{0}^{-4}$, respectively. Overall, these results provide insight into the capability of polymer brush coatings to prevent foulant particles from depositing on a membrane surface.

Funding. The research was funded by grants from the Israel Science Foundation (2018/17) and the Israel Water Authority.

Declaration of interests. The authors report no conflict of interest.

Author ORCIDs.

(D) Avshalom Offner https://orcid.org/0000-0002-0590-2586;

(1) Guy Z. Ramon https://orcid.org/0000-0002-0711-0654.

\section{REFERENCES}

Alexander, S. 1977 Adsorption of chain molecules with a polar head a scaling description. J. Phys. 38 (8), 983-987.

BRENNER, H. 1961 The slow motion of a sphere through a viscous fluid towards a plane surface. Chem. Engng Sci. 16 (3-4), 242-251. 


\section{Interaction of a particle and a polymer brush coating}

FREDRICKSON, G.H. \& Pincus, P. 1991 Drainage of compressed polymer layers: dynamics of a 'squeezed sponge'. Langmuir 7 (4), 786-795.

DE GENNES, P. 1980 Conformations of polymers attached to an interface. Macromolecules 13 (5), 1069-1075.

GoREN, S.L. 1979 The hydrodynamic force resisting the approach of a sphere to a plane permeable wall. J. Colloid Interface Sci. 69 (1), 78-85.

HALPERIN, A.S. 1999 Polymer brushes that resist adsorption of model proteins: design parameters. Langmuir $15,2525-2533$.

Keating, J.J., Imbrogno, J. \& Belfort, G. 2016 Polymer brushes for membrane separations: a review. ACS Appl. Mater. Inter. 8 (42), 28383-28399.

Klein, J., Kumacheva, E., Mahalu, D., Perahla, D. \& Fetters, L.J. 1994 Reduction of frictional forces between solid surfaces bearing polymer brushes. Nature 370, 634-636.

Knox, D.J., Duffy, B.R., McKee, S. \& Wilson, S.K. 2017 Squeeze-film flow between a curved impermeable bearing and a flat porous bed. Phys. Fluids 29 (2), 023101.

Knox, D.J., Wilson, S.K., Duffy, B.R. \& MCKeE, S. 2015 Porous squeeze-film flow. IMA J. Appl. Maths 80 (2), 376-409.

MA, S., Zhang, X., Yu, B. \& Zhou, F. 2019 Brushing up functional materials. NPG Asia Mater. 11 (1), 24.

MiLnER, S.T. 1989 Polymer brushes. Science 251, 905-914.

PotAnin, A.A. \& Russel, W.B. 1995 Hydrodynamic interaction of particles with grafted polymer brushes and applications to rheology of colloidal dispersions. Phys. Rev. E 52 (1), 730-737.

RAMON, G.Z. \& HoEK, E.M.V. 2012 On the enhanced drag force induced by permeation through a filtration membrane. J. Membr. Sci. 392-393, 1-8.

Ramon, G.Z., Huppert, H.E., Lister, J.R. \& Stone, H.A. 2013 On the hydrodynamic interaction between a particle and a permeable surface. Phys. Fluids 25, 073103.

Rubinstein, M. \& Colby, R.H. 2003 Polymer Physics. Oxford University Press.

VEnerus, D.C. 2018 Squeeze flows in liquid films bound by porous disks. J. Fluid Mech. 855, 860-881.

VAN DER WAARDEN, M. 1950 Stabilization of carbon-black dispersions in hydrocarbons. J. Colloid Sci. 5 (4), $317-325$.

Werber, J.R., Osuji, C.O. \& Elimelech, M. 2016 Materials for next-generation desalination and water purification membranes. Nat. Rev. Mater. 1, 16018.

Yan, J., Bockstaller, M.R. \& MAtyjaszewski, K. 2020 Brush-modified materials: control of molecular architecture, assembly behavior, properties and applications. Prog. Polym. Sci. 100, 101180. 